\title{
Understanding the High Strength of L-PBF Metals Using in and ex situ Characterization by TEM and Synchrotron XRD
}

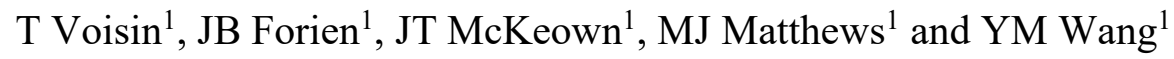 \\ ${ }^{1 .}$ Materials Science Division, Lawrence Livermore National Laboratory, Livermore, CA, USA. \\ * Corresponding author: voisin2@1lnl.gov
}

Additive manufacturing (AM) of metals allows formation of parts with shapes that cannot be made with conventional techniques, or that would require unreasonable time, complexity, and cost to be made otherwise. In addition, selective laser melting, a laser powder bed fusion (L-PBF) technique, allows manufacturing of metals with greatly improved strength compared to their conventional counterparts [14]. L-PBF metals in their as-built state are comprised of non-equilibrium microstructures due to repeated rapid thermos-mechanical cycles encountered during this layer-by-layer process. To capture the unique microstructural features resulting from L-PBF and correlate these features with the unconventional mechanical properties, the as-built material must be investigated at several length scales before, during, and after mechanical testing.

Here, we will review the different techniques we used and their application to investigating L-PBF metals, focusing on our characterization work involving Ti-6Al-4V (wt. \%). Transmission electron microscopy (TEM) plays a major role in characterizing the as-built material from atomic to microscopic scales, together with scanning electron microscopy using electron backscattered diffraction (SEM/EBSD). To correlate small-scale observations to the bulk behavior, we use synchrotron high-energy X-ray diffraction (SXRD) and tensile testing. Both TEM and SXRD can be used in or ex situ, which confers a wide array of experimental observations.

All samples were manufactured with a commercial Concept Laser M2 PBF machine. The laser power and scan speed were varied between 100-250 W and 600-600 mm/s, respectively. The layer thickness was 30 $\mu \mathrm{m}$ and the hatch spacing between $70-105 \mu \mathrm{m}$. All details on processing conditions, experimental set-up, sample preparation procedures, and equipment can be found in our previous publications $[2,5]$.

Figure 1 presents a multiscale experimental approach to characterizing as-built L-PBF Ti-6Al-4V. Figure $1 \mathrm{a}$ is an inverse pole figure obtained by SEM/EBSD. It reveals fine $\alpha$ ' needles in the microstructure, resulting from the martensitic transformation that occurred due to the rapid cooling encountered during L-PBF. TEM (Figure $1 b$ to e) allows identification of the fine nanometer-sized features that can be missed using SEM/EBSD. Very narrow $\alpha$ ' needles can be observed between the larger ones. In addition, a high density of crystalline defects was found in the as-built material, identified as $\langle a\rangle,\langle c+a\rangle$ dislocations and tension, compression twins [5,6]. STEM/EDS was performed (Figure 1f) and revealed chemical heterogeneities, such as segregation of vanadium and iron. Figure $1 \mathrm{~g}$ presents the elastic lattice strain as a function of true stress obtained by SXRD along the loading direction and $90^{\circ}$ away from it (compressive direction). This shows that each family of crystallographic planes presents a different slope and thus a different elastic modulus (between 100 and $120 \mathrm{GPa}$ here). Note the color scale on each curve that represents the changes in full width half maximum (FWHM) as a function of the true stress. The FWHM can be influenced by several factors but one of the most important remains the dislocation density [7]. One can see that the FWHM increases in all planes at the onset of plasticity and after yielding, around 1200-1300 MPa as can be seen on the corresponding stress/strain curve shown in blue. This shows an increase in dislocation density. Since we have observed little to no change in microstructure before and 
after tensile testing (not shown here), this increase in dislocation density is likely responsible for the strain hardening.

In summary, we will present electron microscopy and in situ SXRD techniques, highlighting their importance in understanding the non-equilibrium microstructures of L-PBF metals responsible for their very high strength. In the case of L-PBF Ti-6Al-4V, the fine martensitic structure, together with a high density of dislocations and elemental segregation, is responsible for the high yield strength. In addition, plasticity is still observed after yielding, which explains the several $\%$ of plastic strain even with high flow stress [8].

References:

[1] AM Beese et al., JOM 68(3) (2016), p. 724.

[2] YM Wang et al., Nature Materials 17(1) (2018), p. 63.

[3] M Shamsujjoha et al., Metall and Mat Trans A 49(7) (2018), p. 3011.

[4] L Liu et al., Materials Today 21(4) (2018), p. 354.

[5] T Voisin et al., Materials \& Design 158 (2018), p. 113.

[6] K Kapoor et al., Journal of the Mechanics and Physics of Solids 121 (2018), p. 447.

[7] DW Brown et al., Metall and Mat Trans A 48(12) (2017), p. 6055.

[8] This work was performed under the auspices of the U.S. Department of Energy by Lawrence Livermore National Laboratory under Contract DE-AC52-07NA27344.
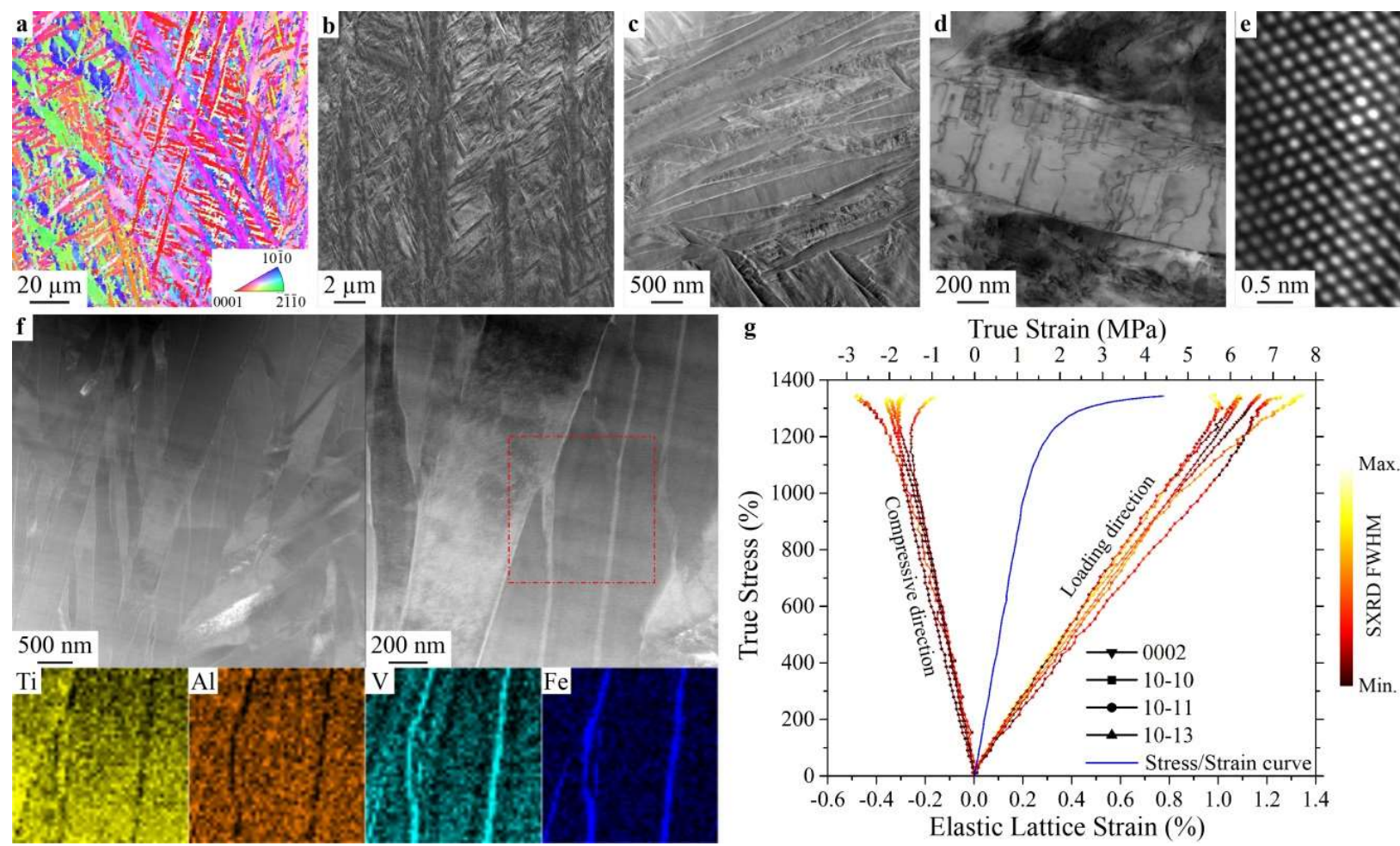

Figure 1. Characterization of L-PBF Ti-6Al-4V after processing and during tensile testing. a) EBSD IPF showing the as-built structure. b) to e) TEM at different magnifications that reveals fine microstructural features. f) STEM/EDS from $\alpha$ ' needles. g) Elastic lattice strain as a function of true stress obtained during in situ SXRD tensile testing. 